b) Deutschland

\title{
Volksbegehren sperrt Parlamentsgesetz? Direkte Demokratie in Thüringen zwischen rechtlich Machbarem und politisch Durchsetzbarem
}

\section{Stefan Storr}

\section{Konkurrenz und Kooperation einer Gesetzgebung durch Parlament und Volk}

Volksbegehren und Volksentscheid sind für die Repräsentativorgane stets eine Herausforderung. Denn die im Parlament vertretenen Parteien müssen sich mit den Anliegen des Volksbegehrens bzw. Volksentscheids auseinandersetzen. Nicht selten werden Volksbegehren von Oppositionsparteien unterstützt, aber auch genutzt, um ein Gesetz gegen die regierungsbildende Mehrheit zu schaffen - und damit zugleich der Regierungsmehrheit eine politische Niederlage beizubringen. Liegt bei einer erfolgreichen Volksgesetzgebung der Vorwurf an die regierungsbildende Mehrheit, die sich dem Volksbegehren nicht angeschlossen hat, nicht nahe, sie habe - wenn auch nicht de jure, doch aber de facto - in dieser Frage die Mehrheit der Bevölkerung nicht vertreten, indem sie ,am Volk vorbei“ gehandelt hat oder untätig geblieben ist?

Von Verfassungs wegen ist es zwingend, beide Wege der Gesetzgebung schon wegen ihrer unterschiedlichen Verfahrensvoraussetzungen demokratischer Willensbildung strikt zu unterscheiden; in der Verfassungswirklichkeit aber sind Volks- und parlamentarische Gesetzgebung aufeinander bezogen: Die Volksgesetzgebung hat eine Ergänzungsfunktion ${ }^{1}$ neben der überwiegend parlamentarischen Gesetzgebung; dem Parlament kommt die Aufgabe zu, das Verfahren der Volksgesetzgebung zu begleiten, ggfs. darauf zu reagieren (vgl. nur Art. 82 Abs. 7 ThürVerf.). Es ist diese antagonistische Konkurrenz- und Kooperationssituation, die das eigentümliche Gegen- und Miteinander von Volks- und parlamentarischer Gesetzgebung ausmacht. In den Jahren 2007 bis 2009 kam es in Thüringen zu einer besonders prekären Konkurrenzsituation von parlamentarischer und Volksgesetzgebung, die sich letztlich in einer Kooperation auflöste.

1 P.M. Huber, Ergebnisprotokoll und Wortprotokoll, 67. Sitzung des Innenausschusses des Thüringer Landtags am 13. Januar 2009, S. 27. 


\section{Der Konflikt: Ein Gesetz des Landtags , überholt“ das Volksbegehren}

Am 31. Juli 2007 war in Thüringen das Volksbegehren „Mehr Demokratie in Thüringer Kommunen" mit einer Unterschriftensammlung gestartet. ${ }^{2}$ Gegenstand des Volksbegehrens war eine Erweiterung plebiszitärer Mitwirkungsrechte auf kommunaler Ebene durch Änderung der Thüringer Kommunalordnung. Der Bürgerantrag sollte durch einen Einwohnerantrag ersetzt und die Voraussetzungsquoren auf $1 \%$, höchstens aber 300 Einwohner (bisher 10\%) herabgesetzt werden. Auch Personen, die das 14. Lebensjahr vollendet haben, sollten den Einwohnerantrag unterstützen können. Die erforderlichen Quoren für ein Bürgerbegehren sollten herabgesetzt werden (Zustimmung von mindestens 7\% der Bürger und höchstens 7.000 Stimmberechtigten in vier Monaten; bisher 20\%). Regelungen zur Durchführung der Sammlung sollte es nicht geben und damit auch die freie Sammlung ,auf der Straße“ zulässig sein. Der Themenausschlusskatalog (Aufzählung der für ein Bürgerbegehren ausgeschlossenen Themen) sollte neu gefasst werden. Für Landkreise sollten entsprechende Regelungen gelten. ${ }^{3}$ Auf Einzelheiten ist an dieser Stelle nicht einzugehen.

12.862 Stimmberechtigte unterzeichneten den Antrag auf Durchführung des Volksbegehrens, 5.000 wären erforderlich gewesen. Am 21. Januar 2008 machte die Präsidentin des Thüringer Landtags den zulässigen Antrag des Volksbegehrens „Mehr Demokratie in Thüringer Kommunen“ und die Sammlungsfrist bekannt, ${ }^{4}$ die vom 20. März 2008 bis zum 19. Juli 2008 lief. Über 235.530 Stimmberechtigte (von 195.325 erforderlichen) unterstützten das Volksbegehren. Am 4. August 2008 wurden die Unterschriften von den Initiatoren des Volksbegehrens dem Innenministerium zur Prüfung vorgelegt. Mit Bescheid vom 23. Oktober 2008 stellte die Präsidentin des Landtags das Zustandekommen des Volksbegehrens fest. ${ }^{5}$

Doch bereits am 2. Mai 2008, also während der Sammlungsfrist, hatte die Fraktion der CDU im Thüringer Landtag den Entwurf für ein Gesetz zur Stärkung des bürgerschaftlichen Engagements und zur verbesserten Teilhabe an kommunalen Entscheidungsprozessen eingebracht. ${ }^{6}$ Auch dieser sah einen Einwohnerantrag vor, aber nur für Volljährige und - in einem ersten Vorschlag - ein erhöhtes Stimmrecht für Eltern. Für den Bürgerantrag sollten die Quoren auf 5 bis $10 \%$ abgesenkt wer-

2 Zum Hintergrund: P. Neumann, Sachunmittelbare Demokratie, 2009, S. 779 f.

3 Gesetzentwurf nach Artikel 82 der Verfassung des Freistaats Thüringen - Volksbegehren „Mehr Demokratie in Thüringer Kommunen“; Entwurf eines Gesetzes zur Änderung der Thüringer Kommunalordnung (Gesetz für mehr direkte Demokratie in Thüringer Kommunen), LT-Drs. $4 / 4550$ vom 23. Oktober 2008.

$4 \S 13$ Abs. 1 ThürBVVG: ThürGVB1. vom 23. Januar 2008, S. 4.

$5 \S 17$ Abs. 2 und 3 ThürBVVG: mit Bescheid der Präsidentin des Landtags vom 23. Oktober 2008.

6 Fraktion der CDU im Thüringer Landtag, Thüringer Gesetz zur Stärkung des bürgerschaftlichen Engagements und zur verbesserten Teilhabe an kommunalen Entscheidungsprozessen, LT-Drs. 4/4084 vom 2.5.2008 und Änderungsantrag der Fraktion der CDU im Thüringer Landtag zum Gesetzentwurf der Fraktion der CDU, LT-Drs. 4/4084 vom 25.9.2008, Vorlage 4/2361; Beschlussempfehlung Innenausschuss LT-Drs. 4/4483 vom 2.10.2008. 
den; die Form der Sammlung aber wurde auf eine Eintragung in amtlich ausgelegte Eintragungslisten beschränkt („Amtsstubensammlung“). Der Themenausschlusskatalog wurde ebenfalls geändert. Auch auf Landkreisebene sollten direktdemokratische Rechte und Verfahren eingeführt werden. Die Einzelheiten sollen auch hier nicht im Vordergrund stehen. Wichtiger ist der Verfahrensablauf: Der CDU-Entwurf wurde im Thüringer Landtag am 8. Mai 2008 in erster Beratung und am 9. Oktober 2008 in zweiter Beratung debattiert und sodann beschlossen. Am 9. Oktober 2008 wurde das Gesetz von der Präsidentin des Landtags ausgefertigt und am 17. Oktober 2008 im Gesetz- und Verordnungsblatt verkündet. Am 18. Oktober 2008 trat es in Kraft. ${ }^{7}$

Das Änderungsgesetz des Landtags war als Artikelgesetz verfasst; u.a. wurde die Thüringer Kommunalordnung geändert. Diese Änderungen waren aber auf das Volksbegehren nicht abgestimmt. Das Volksbegehren hatte Änderungen der Thüringer Kommunalordnung vorgeschlagen, die sich auf den Rechtsstand und den Gesamttext, wie er sich nach der letzten Gesetzesänderung am 23. Dezember 2005 darstellte, bezogen. Durch das Landtagsgesetz vom 9. Oktober 2008 verschob sich nun aber die ursprüngliche Systematik der Normen der Kommunalordnung. Ein Volksentscheid über den Gesetzentwurf des Volksbegehrens würde also nicht nur über eine Fassung der Thüringer Kommunalordnung erfolgen, die zum Zeitpunkt der Sammlung des Volksbegehrens nicht gegolten hat, sondern auch, wenn der Volksentscheid Erfolg haben sollte, zu intranormativen Verwerfungen führen. Damit aber entstand die verfassungsrechtliche Frage, wie sich beide Gesetzgebungsverfahren zueinander verhalten, insbesondere ob der Landtag ein das Volksbegehren „überholendes Gesetz“ erlassen durfte und zu welcher Gesetzeslage es bei einem möglicherweise erfolgreichen Volksentscheid kommen würde. Im Verfahren der Beratung des Volksbegehrens führte der Innenausschuss des Landtags am 13. Februar 2009 eine öffentliche Anhörung durch, in der es im Wesentlichen um diese Fragen ging.

\section{Das Problem: Ist eine „,überholende Gesetzgebung “ des Landtags zulässig?}

\section{a) Parlamentsgesetzgebung und Volksgesetzgebung als parallele Verfahren}

Die Vertreter des Volksbegehrens suchten nach einer juristischen Begründung, nach der es dem Landtag untersagt sein soll, noch vor der amtlichen Feststellung des Volksbegehrens ein Gesetz zu beschließen, das in der Sache dem Volksbegehren nicht entspricht. Sperrregeln gibt es in der Thüringer Kommunalordnung für Bürgerbegehren - nach der Feststellung der Zulässigkeit des Bürgerbegehrens darf eine dem Begehren entgegenstehende Entscheidung der Gemeindeorgane nicht mehr getroffen oder mit dem Vollzug einer derartigen Entscheidung nicht mehr begonnen

7 Thüringer Gesetz zur Stärkung des bürgerschaftlichen Engagements und zur verbesserten Teilhabe an kommunalen Entscheidungsprozessen vom 9.10.2008, ThürGVB1. 2008, S. 353. 
werden $^{8}$ - die Thüringer Verfassung aber kennt kein Beratungs- oder Gesetzgebungsverbot des Landtags während eines laufenden Volksbegehrens. Vielmehr räumt die Verfassung dem Landtag die Möglichkeit ein, dem Volk zusätzlich einen eigenen Gesetzentwurf zur Entscheidung vorzulegen. Eine Konkurrenz zwischen Volksbegehren und Landtag ist also nicht unzulässig, sondern - wie Art. 82 Abs. 7 ThürVerf. zeigt - von der Verfassung akzeptiert. Denn das Denken in Alternativen und der Wettstreit zwischen politischen Konzepten sind einer Demokratie immanent. ${ }^{9}$

Die Gesetzgebung durch den Landtag und im Wege des Volksbegehrens/Volksentscheids ist insofern funktional äquivalent, als beide Gesetzgeber parallel Gesetze erlassen können. Im Anhörungsverfahren ist vorgetragen worden, aus Art. 82 Abs. 7 S. 2 ThürVerf. ergebe sich, dass der Landtag im Fall eines laufenden Volksgesetzgebungsverfahrens nur die Möglichkeit habe, dem Volk einen eigenen Gesetzentwurf vorzulegen, wenn er dem Volksbegehren nicht folgen will. ${ }^{10}$ Das trifft nicht zu. Art. 82 Abs. 7 ThürVerf. regelt einen Fall konkurrierender Gesetzgebung, schliesst andere Varianten aber nicht aus. Im vorliegenden Fall findet Art. 87 Abs. 7 S. 2 ThürVerf. schon keine Anwendung. Die Bestimmung bezieht sich auf den Verfahrensstand nach Feststellung des Zustandekommens des Volksbegehrens durch die Präsidentin des Landtags (Art. 82 Abs. 7 S. 2 ThürVerf.). Dann hat der Landtag das Volksbegehren innerhalb von sechs Monaten abschließend zu behandeln. Das Gesetz des Landtags ist am 18. Oktober 2008 in Kraft getreten; die Präsidentin des Landtags hat das Zustandekommen des Volksbegehrens aber erst fünf Tage später festgestellt. ${ }^{11}$

Darüber hinaus ist Art. 82 Abs. 7 S. 2 ThürVerf. als eine Vorschrift konzipiert, die nicht die Rechte des Landtags beschränken, sondern erweitern soll. Der Landtag soll „zusätzlich“ „auch“ einen eigenen Gesetzesentwurf präsentieren können. Eine Beschränkung der parlamentarischen Gesetzgebungsbefugnis hätte einer ausdrücklichen verfassungsrechtlichen Anordnung bedurft. Denn ausweislich Art. 48 ThürVerf. ist der Landtag das vom Volk gewählte oberste Organ der demokratischen Willensbildung und übt gesetzgebende Gewalt aus. „Oberstes Organ demokratischer Willensbildung“ bedeutet nicht, dass ihm nur eine „,sozial hohe Stellung, die höchste Stellung im Bereich der politischen Willensbildung" 12 zukommt, sondern richtet den Landtag als die Volksvertretung ein und als das Gremium, in dem die politische Auseinandersetzung institutionalisiert ist. ${ }^{13}$ Das bedeutet weder, dass Parlamentsgesetze den Volksgesetzen gegenüber höherrangig sind, noch dass dem Volk eine gesetzgebende Gewalt nur zukäme, wenn das Parlament von seiner Gesetzgebungs-

$8 \S 17$ Abs. 6 ThürKO a.F.; $\S 17$ Abs. 5 ThürKO n.F.

9 S. Storr/M. Koch, Das Plebiszit in Thüringen - eine Erfolgsgeschichte?, in: ThürVB1. 2009, S. $5(8)$.

10 H. Meyer, Ergebnisprotokoll und Wortprotokoll, 67. Sitzung des Innenausschusses des Thüringer Landtags am 13. Januar 2009, S. 73.

11 Vgl. a. Huber, Ergebnisprotokoll (Fn. 1), S. 72.

12 So aber Meyer, Ergebnisprotokoll (Fn. 10), S. 72.

13 S. Storr, Staats- und Verfassungsrecht, 1998, S. 153. 
befugnis keinen Gebrauch macht (was Art. 82 ThürVerf. ja bereits ausschließt), es bedeutet aber, dass die Kompetenz des Landtags zur demokratischen Willensbildung grundsätzlich besteht. Das wiederum heißt, dass jede Beschränkung dieser Kompetenz einer ausdrücklichen verfassungsrechtlichen Regelung bedarf. Der Thüringer Verfassungsgerichtshof spricht von einer „Prävalenz“ des parlamentarischen Gesetzgebers gegenüber dem plebiszitären Gesetzgeber ${ }^{14}$ und meint damit ein RegelAusnahme-Verhältnis, ${ }^{15}$ dem die Überlegung zugrunde liegt, dass es zunächst Aufgabe des Landtags sein soll, Gesetze für die Allgemeinheit zu beschließen; die Volksgesetzgebung hingegen kann schon aus praktischen Gründen nur auf einzelne Angelegenheiten beschränkt sein, ihr kann nur eine Ergänzungsfunktion zur parlamentarischen Gesetzgebung zukommen.

Für dieses Ergebnis spricht auch $\S 19$ Abs. 2 S. 1 ThürBVVG. Verfassungsrechtliche Bedenken gegen diese Vorschrift hier dahingestellt, ${ }^{16}$ regelt sie den Fall, dass der Landtag den begehrten Gesetzentwurf in veränderter Form annimmt, die jedoch dem Grundanliegen des Volksbegehrens entspricht. Dann kann der Landtag auf entsprechenden Antrag der Vertrauensperson die Erledigung des Volksbegehrens feststellen. Damit setzt die Vorschrift voraus, dass der Landtag neben dem Volksgesetzgebungsverfahren ein Gesetzgebungsverfahren betreiben kann.

\section{b) Beschränkung der Parlamentsgesetzgebung durch den Grundsatz der Organtreue?}

Die Hauptargumentationslinie der Befürworter eines Verbots überholender Parlamentsgesetze geht dahin, die überholende Gesetzgebung sei unredlich ${ }^{17}$ und verstoße gegen den Verfassungsgrundsatz der Organtreue, der dem parlamentarischen Gesetzgeber eine Pflicht zur Rücksichtnahme und Kooperation auferlege. ${ }^{18}$ Aus der Organtreue sollen Verpflichtungen hergeleitet werden, welche die Kompetenzen des jeweils anderen Organs beschneiden; ein unter Verstoß gegen die Organtreue beschlossenes Gesetz soll verfassungswidrig sein.

Die Landesverfassungsgerichte von Hamburg und Sachsen ${ }^{19}$ sind davon ausgegangen, dass die für das Grundgesetz als ungeschriebener Rechtsgrundsatz entwickelte Organtreue auch Parlament und Volksgesetzgeber verpflichtet. Der Thür-

14 ThürVerfGH ThürVB1. 2002, 31 (37).

15 Kritisch $F$. Wittreck, Direkte Demokratie und Verfassungsgerichtsbarkeit, in: JöR n.F. 53 (2005), 111 (179).

16 Dazu unter 5.

17 Vgl. Abg. R. Hahnemann, Ergebnisprotokoll und Wortprotokoll, 67. Sitzung des Innenausschusses des Thüringer Landtags am 13. Januar 2009, S. 33: „politische Moral“; Sachverst. K. Hahnzog, ebenda, S. 44: „Respekt vor der Volksgesetzgebung“; Sachverst. G. Kirchgässner, ebenda, S. 88: „unfair“.

18 L. Hasse, Volksbegehren in guter Verfassung!, in: ThürVB1. 2009, S. 73 (75).

19 VerfG Hamburg vom 30. November 2004, HVerfG 6/04 (NVwZ 2005, 685); SächsVerfGH vom 17. Juli 1998 - Vf. 32-I-98 (SächsVBl. 1998, 216). 
VerfGH hat noch nicht darüber entschieden, ob dieser Grundsatz auch im Verhältnis zwischen Thüringer Landtag und Volksgesetzgeber gilt.

Daran bestehen grundlegende Zweifel. Denn das Verhältnis von Verfassungsorganen zueinander ist zunächst einmal in der Verfassung geregelt, für Landtag und Volksbegehren zentral in Art. 45, 48 und 82 ThürVerf. In diesen Vorschriften sind Volks- und Parlamentsgesetzgebung als konkurrierende Gesetzgebungsverfahren angelegt. Es gibt keine Vorschrift, die das eine oder andere Gesetzgebungsverfahren wegen einer Konkurrenzsituation beschränkt. Folglich können beide Verfahren auch parallel ablaufen. Gerade diese verfassungsimmanente Verfahrenskonkurrenz steht der Annahme eines Verfassungsgebots der Organtreue grundsätzlich entgegen, wenn dieses eine Kompetenzbeschränkung des Landtags zur Folge haben soll. ${ }^{20}$ Diese Verfahrenskonkurrenz ist wegen ihres Konfliktpotentials nicht negativ zu bewerten, sondern im demokratischen Sinne zu begrüßen, weil eine breite Diskussion über Gesetzesinitiativen zugelassen wird und Abgeordnete und Bürger in die Gesetzgebungsprozesse eingebunden werden können. Allein, dass Volksgesetzgebung zeitaufwändiger ist als parlamentarische Gesetzgebung, ändert daran nichts. Die Schwerfälligkeit liegt in der Natur der Sache, weil die Volksgesetzgebung die Stadien von Volksbegehren und Volksentscheid durchlaufen muss.

Aus der verfassungsrechtlichen Gewährleistung von Volksbegehren und Volksentscheid folgt aber, dass die Staatsgewalt die Effektivität dieser Volksgesetzgebungsrechte zu gewährleisten hat. ${ }^{21}$ Weil das Parlament selbst gesetzgebend tätig werden darf, kann das Gebot der Organtreue wegen der Konkurrenz zum Volksbegehren allenfalls in Ausnahmefällen Bedeutung haben und das Parlament in die Pflicht nehmen, die Ausübung dieser Volksgesetzgebungsrechte nicht missbräuchlich zu behindern oder zu beschränken.

Im Sinne einer engen Interpretation ist auch das Urteil des VerfG Hamburg 22 zu verstehen. Danach bedeutet der Grundsatz der Organtreue, dass sich die Staatsorgane im Verhältnis zueinander so zu verhalten haben, dass sie ihre verfassungsrechtlichen Zuständigkeiten verantwortlich und gewissenhaft, frei von Zeitnot und Pressionen ausüben können. Der Grundsatz der Organtreue ,führt aber nicht dazu, dass die Kompetenzen des einen Verfassungsorgans durch das andere aufgehoben oder eingeschränkt werden." Auch der SächsVerfGH ${ }^{23}$ begreift die Organtreue als eine Art verfassungsrechtliche „Notbremse“: Danach obliegt es dem Landtag, auf das Volk bei seinen Gesetzgebungsverfahren auch insoweit in angemessener Weise Rücksicht zu nehmen, als es sich zur Durchführung eines Volksantrages oder Volksbegehrens organisiert und damit in Konkurrenz zu parlamentarischen Kompetenzen tritt. Die Zurückstellung eigener Gesetzgebungsvorhaben kann vom Landtag jedoch ,allenfalls ganz ausnahmsweise verlangt werden". Das Gebot der Organtreue - so der ThürVerfGH in anderem Zusammenhang - verschafft keinem Verfassungsorgan das Recht, unter Berufung auf eine Treuepflicht in den Kernbereich der Kompetenzen

20 So aber Meyer, Ergebnisprotokoll (Fn. 10), S. 78.

21 BayVerfGH vom 6. Mai 2005 - 21/IX/05 (NVwZ-RR 2005, 754).

22 VerfG Hamburg, HVerfG 6/04 (Fn. 19).

23 SächsVerfGH, Vf. 32-I-98 (Fn. 19). 
eines anderen Verfassungsorgans einzudringen. ${ }^{24}$ Deshalb liegt ein Missbrauch nicht schon dann vor, dass der Landtag ein Gesetz verabschiedet, das dem ,eigentlichen Anliegen“" des Volksbegehrens widerspricht. ${ }^{25}$

Keinesfalls aber kann der Grundsatz der Organtreue zur Anwendung gelangen, wenn das Zustandekommen des Volksbegehrens noch nicht festgestellt ist. Denn bis zur Feststellung des Zustandekommens besteht kein verfassungsrechtliches Rechtsverhältnis zwischen dem Volksbegehren und dem Parlament, und das Volksbegehren ist auch noch kein Verfassungsorgan. ${ }^{26}$ Auf den zulässigen Antrag auf Durchführung eines Volksbegehrens, für den bereits 5.000 Unterstützer genügen, ${ }^{27} \mathrm{kann}$ schon deshalb nicht abgestellt werden, weil zu diesem Zeitpunkt für den Landtag oder die Landtagsmehrheit nicht erkennbar ist, ob dieser Antrag auf die erforderliche Resonanz in der Bevölkerung stoßen wird, die eine Beschränkung des parlamentarischen Gesetzgebungsrechts rechtfertigen würde. ${ }^{28}$ Bezeichnenderweise ging es im Hamburger Fall um die Berücksichtigung eines bereits zustande gekommenen Volksentscheids, und ausdrücklich führt der SächsVerfGH in seinen Urteilsgründen aus, dass der Volksentscheid kurz vor seinem Abschluss stehen muss, ,zumindest aber bereits ein Volksbegehren erfolgreich abgeschlossen "29 sein muss. Das ist schon deshalb richtig, weil es vor der amtlichen Feststellung des Zustandekommens des Volksbegehrens keinen Zeitpunkt gibt, zu dem rechtssicher gesagt werden kann, dass das Volksbegehren wirksam ist. ${ }^{30}$

\section{Das Zukunftsszenario: Die Gesetzeslage im Fall eines Zustandekommens des Volksentscheids}

Ein erfolgreicher Volksentscheid hätte normative Brüche in der Thüringer Kommunalordnung zur Folge gehabt. Diese hätten daraus resultiert, dass der Thüringer Landtag mit Verabschiedung des Gesetzes zur Stärkung des bürgerschaftlichen Engagements und zur verbesserten Teilhabe an kommunalen Entscheidungsprozessen vom 9. Oktober 2008 die Vorschriften über Bürgerantrag und Bürgerbegehren abgeändert hatte, der Entwurf des Volksbegehrens aber auf die Rechtslage in der Fassung vom 23. Dezember 2005 Bezug genommen hatte.

Diese Konfliktlage wäre nicht dadurch aufgelöst worden, dass das überholende Gesetz des Landtags mit Feststellung der Wirksamkeit des Volksbegehrens oder des Zustandekommens des Volksentscheids unisono und zur Gänze nichtig und der Rechtszustand von 2005 wiederhergestellt worden wäre bzw. daran hätte angeknüpft werden können. Einen derartigen Vorrang der Volksgesetzgebung vor der parla-

24 ThürVerfGH vom 19. Dezember 2008 - VerfGH 35/07 (DVB1. 2009, 245).

25 Storr/Koch, Plebiszit (Fn. 9), S. 8.

26 P.M. Huber, Aktuelle Fragen der direkten Demokratie in Thüringen, Gutachten 2009, S. 38.

$27 \S \S 10,13$ ThürBVVG.

28 Huber, Ergebnisprotokoll (Fn. 1), S. 28.

29 SächsVerfGH vom 17. Juli 1998 - Vf. 32-I-98.

30 Huber, Aktuelle Fragen (Fn. 26), S. 38. 
mentarischen Gesetzgebung kennt die Verfassung nicht. Sicher wird das Gesetzgebungsverfahren des Landtags häufig einfacher und der Landtag agiler sein; ${ }^{31}$ darauf kommt es aber nicht an, sondern nur, ob es eine Rechtsnorm gibt, die diese Rechtsfolge anordnet. Das ist nicht der Fall.

Auch das Gesetz für mehr direkte Demokratie in Thüringen selbst konnte, als es später durch Landtagsbeschluss und Verkündung im Gesetzblatt in Kraft trat, das vom Landtag zuvor beschlossene Gesetz, das bereits in Kraft getreten war, nicht zur Gänze aufheben und an den Rechtszustand vor Inkrafttreten des Landtagsgesetzes anknüpfen. Eine entsprechende Anordnung fehlte bereits im Gesetzentwurf. Zwar nahm das Volksbegehren in seinem Entwurf im Vorspruch auf die Gesetzeslage zu einem bestimmten Zeitpunkt Bezug (,Die Thüringer Kommunalordnung in der Fassung vom 28. Januar 2003 [GVBl. S. 41], zuletzt geändert durch Artikel 5 des Gesetzes vom 23. Dezember 2005 [GVBl. S. 446], wird wie folgt geändert:"); durch einen solchen Vorspruch kann ein im Wege der Volksabstimmung zustande gekommenes Gesetz ein „überholendes Landtagsgesetz“ aber nicht aufheben. Denn ein Vorspruch ist nicht Gegenstand eines Gesetzesvorschlags und wird auch nicht Gesetz.

Vielmehr gilt in solchen Fällen, dass entstehende Normenkonflikte durch Interpretation, d. h. grundsätzlich nach der Konfliktlösungsregel „lex posterior derogat legi priori““ aufzulösen sind. Mit Inkrafttreten des volksbeschlossenen Gesetzes wären Teile des überholenden Landtagsgesetzes vom 9. Oktober 2008 aufgehoben worden, andere Teile wären in Kraft geblieben. Insgesamt wäre es zu einem Bruch in der Gesetzessystematik gekommen, die zu bereinigen erneute Aufgabe des Gesetzgebers gewesen wäre. Hierzu hätte in besonderen Fällen eine Neubekanntmachung veranlasst werden können, systematische Gesetzesbrüche hätten aber einer neuen Regelung bedurft. Diese verzwickte Konstellation zeigt die besondere Sorgfalt, mit der die Initiatoren eines Volksbegehrens ihren Gesetzentwurf formulieren müssen, um das Volksbegehren bei einer überholenden Gesetzesänderung durch den Landtag zum vollen Erfolg führen zu können.

\section{Die politische Lösung: Der große Kompromiss}

In rechtlicher Hinsicht war das Landtagsgesetz vom 8. Oktober 2008 daher trotz laufenden Volksgesetzgebungsverfahrens wirksam. Durchgesetzt hat sich aber eine politische Lösung. Die Vertreter des Volksbegehrens und die diese unterstützenden Oppositionsfraktionen hatten am 22. Dezember 2008 Klagen gegen das Landtagsgesetz vom 9. Oktober 2008 beim ThürVerfGH eingereicht und den Druck auf den politischen Gegner erhöht. Wie die Ausführungen aber gezeigt haben, war ein Erfolg

31 F. Wittreck, Direkte Demokratie vor Gericht oder: Demokratie und Verfassungsgerichtsbarkeit - Ein gestörtes Verhältnis?, in: H.K. Heußner/O. Jung (Hrsg.), Mehr direkte Demokratie wagen, 2. Aufl. 2009, S. 397 (403). 
vor dem Verfassungsgerichtshof mehr als zweifelhaft. Schließlich einigten sich die Vertreter des Volksbegehrens und die Oppositionsfraktionen mit der CDU-Fraktion.

Am 1. April 2009 brachten die Fraktionen der CDU, DIE LINKE und der SPD einen Entwurf zu einem Begleitgesetz zum Gesetz zur Änderung der Thüringer Kommunalordnung ein. ${ }^{32}$ Gegenstand des Gesetzentwurfs war eine weitgehende Übernahme der Regelungen des Volksbegehrens, allerdings unter Änderung der Gesetzessystematik und unter Einfügung von zwei Varianten der Sammlungsform, einerseits die freie Sammlung ( $\$ 17$ a ThürKO 2009) mit Quorumsanforderungen von $7 \%$, wobei die Anforderungen an die Sammlung - anders als im Volksbegehrensentwurf - im Gesetz näher geregelt wurden, andererseits - als Alternative - die Sammlung durch Eintragung in amtlich ausgelegte Eintragungslisten $(\S 17 \mathrm{~b}$ ThürKO 2009) mit einem Quorum von $6 \%$ der Stimmberechtigten. Bereits am 3. April 2009 wurde im Landtag kurz hintereinander über zwei Gesetzentwürfe abgestimmt: ${ }^{33}$ Zunächst über den Entwurf des Volksbegehrens, der fraktionsübergreifend mit großer Mehrheit angenommen und als Viertes Gesetz zur Änderung der Thüringer Kommunalordnung im Gesetz- und Verordnungsblatt vom 22. April $2009^{34}$ veröffentlicht wurde und einen Tag später in Kraft trat; sodann über den fraktionsübergreifenden Kompromissvorschlag vom 1. April, der als Fünftes Gesetz zur Änderung der Thüringer Kommunalordnung im Gesetz- und Verordnungsblatt vom 23. April 2009 veröffentlicht wurde und vierzehn Tage später in Kraft getreten ist. ${ }^{35}$ Mit dem Fünften Gesetz zur Änderung der Thüringer Kommunalordnung wurde das Vierte Gesetz zur Änderung der Thüringer Kommunalordnung formal wieder aufgehoben, in der Sache wurden dessen Regelungen aber weitgehend beibehalten, zum Teil mit Vorschriften über die freie Sammlung und Eintragung in amtlich ausgelegte Eintragungslisten aber modifiziert.

Eine sicherlich umständliche Vorgehensweise, die aber zum Ziel hatte, einen Volksentscheid trotz Modifizierung des Gesetzentwurfs wie er dem Volksbegehren zugrunde lag, zu vermeiden. Der Volksentscheid konnte sich von Verfassungs wegen nur dann erledigen, wenn der Landtag dem Volksbegehren vollumfänglich entsprach (Art. 87 Abs. 7 S. 1 ThürVerf.). Darüber hinaus regelt $§ 19$ Abs. 2 ThürBVVG zwar, dass die Vertrauensperson einen Antrag auf Feststellung der Erledigung des Volksbegehrens stellen kann, wenn der Landtag den begehrten Gesetzentwurf in veränderter Form annimmt, damit aber dem „Grundanliegen des Volksbegehrens“ entspricht. Diese Vorschrift bedarf aber einer verfassungskonformen Interpretation, weil nach Art. 87 Abs. 7 S. 1 ThürVerf. ein Volksentscheid bereits dann stattzufinden hat, wenn der Landtag einem zulässigen Volksbegehren „nicht entspricht“. Dieser

32 Gesetzentwurf der Fraktionen der CDU, DIE LINKE und der SPD Begleitgesetz zum Gesetz zur Änderung der Thüringer Kommunalordnung (Gesetz für mehr direkte Demokratie in Thüringer Kommunen) - Volksbegehrens-Begleitgesetz, LT.-Drs. 4/5062 vom 1.4.2009.

33 Thüringer Landtag, Plenarprotokoll 4/105 vom 3.4.2009, 105. Sitzung.

34 Viertes Gesetz zur Änderung der Thüringer Kommunalordnung (Gesetz für mehr direkte Demokratie in Thüringer Kommunen) vom 8.4.2009, GVB1. S. 320.

35 Begleitgesetz zum Gesetz zur Änderung der Thüringer Kommunalordnung (Gesetz für mehr direkte Demokratie in Thüringer Kommunen) - Volksbegehrens-Begleitgesetz, Fünftes Gesetz zur Änderung der Thüringer Kommunalordnung vom 8.4.2009, GVB1. S. 345. 
Automatismus dient dem Schutz der Volksgesetzgebung, weil die das Volksbegehren unterstützenden Stimmberechtigten aus unterschiedlichen Gründen das Volksbegehren unterstützt haben können, die Unterstützer selbst aber mit den politischen Kräften im Landtag nicht verhandeln können. Jede Modifizierung des Gesetzentwurfs, der dem Volksbegehren zugrunde liegt, durch die Vertrauensperson ohne entsprechende Legitimation durch die Unterstützer des Volksbegehrens widerspricht dem Sinn des Volksbegehrens als Mitwirkung „des Volkes“ an der Staatswillensbildung. Abweichungen vom Gesetzentwurf des Volksbegehrens verfälschen den Willen der Unterzeichner und sind deshalb grundsätzlich unzulässig. ${ }^{36}$ Deshalb muss gelten, dass die Vertrauensperson grundsätzlich kein Jota vom Volksbegehren abweichen kann. ${ }^{37}$ Das gilt auch für den Landtag, der nach Art. 82 Abs. 7 S. 1 ThürVerf. über das Volksbegehren Beschluss zu fassen hat. Durch die Einfügung der Amtsstubensammlung hat der Landtag keinesfalls dem Volksbegehren entsprochen, auch nicht durch weitergehende Regelungen zur freien Sammlung (§ 17 a Abs. 2 ThürKO 2009). Deshalb schien den Beteiligten der politische Deal am erfolgversprechendsten: Der Landtag sollte den Gesetzentwurf des Volksbegehrens annehmen und dieser sollte Gesetz werden. Sodann sollte ein neues Begleitgesetz in Kraft gesetzt werden, das die Rechtslage bereinigt, weitere Änderungen, welche die CDU im Kompromiss durchgesetzt hat, aufnimmt, aber dennoch dem Volksbegehren in der Sache entspricht. Zum Deal gehörte auch, dass die Kläger ihre Klagen vor dem ThürVerfGH zurücknehmen sollten.

Rückblickend zeigt dieses politische Gefecht einmal mehr, dass einer Volksgesetzgebung zwar „nur“ eine Ergänzungsfunktion zur repräsentativen Demokratie zukommen kann; wenn dieser Weg aber beschritten wird, können die Repräsentativorgane - insbesondere die regierende Mehrheit - vor erhebliche politische Herausforderungen gestellt sein. Die Verfassung lässt eine Konkurrenz von Parlamentsgesetzgebung und Volksgesetzgebung zu und befeuert damit den politischen Wettbewerb. Trotz der Konkurrenz von Landtagsmehrheit auf der einen und Volksbegehrensvertretern sowie hier der Landtagsopposition auf der anderen Seite kam es am Ende zu einer übergreifenden Kooperation der maßgebenden politischen Kräfte.

36 Storr/Koch, Plebiszit (Fn. 9), S. 10; vgl. zu einer engen Interpretation auch: ThürVerfGH vom 19.9.2001, VerfGH 4/01, Gliederungspunkt B.II.21.

37 Storr, Verfassungsrecht (Fn. 13), S. 277; Neumann, Demokratie (Fn. 2), S. 625. 